\title{
Ist eine Allgemeine Kommunikationswissenschaft möglich?
}

Eine Autopolemik

Manfred Rühl

Das Wissenschaftssystem der Weltgesellschaft erneuert das von ibm in der Vergangenheit produzierte Wissen durch eine sich selbst kontrollierende Wissensreproduktion. Zur Rekonstruktion einer Theorie der Allgemeinen Kommunikationswissenschaft sind bewabrte, also nicht vergessene Kommunikationstheorien wissenschaftskritisch zu bearbeiten. Es gibt keine Urformel für den Zugang zur menschlichen Kommunikation. Kommunikationswissenschaftliche Theorien sind in Orientierung am Wandel vor einem sozialwissenschaftlichen Wissenshorizont zu bearbeiten. Dazu wird hier die System/Mitwelt-Theorie als Erkenntnishilfe und der empirisch-vergleichende Funktionalismus als Methode gewählt. Medientheoretische und medienpraktische Probleme werden auf ibre kommunikationswissenschaftliche Brauchbarkeit befragt. Der Beitrag diskutiert zur Rekonstruktion einer Theorie der Allgemeinen Kommunikationswissenschaft zwei Untersuchungsbedingungen: (1) Veraltete Theorien werden mit Gaston Bachelard als Erkenntnishindernisse identifiziert und mit Thomas Kubn zur Aufräumarbeit empfoblen. (2) Neue kommunikationswissenschaftliche Erkenntnisse können, so Kenneth Burke, durch inkongruente Perspektiven erschlossen werden. Die Analyse wird exemplarisch vorgenommen.

Keywords: Kommunikationswissenschaft, empirische Medienforschung, sozialwissenschaftlicher Theorienpluralismus, Epistemologie, Methodologie, Grundlagenforschung, angewandte Forschung, Erkenntnishindernisse, inkongruente Perspektive

\section{Einleitung}

Jahrestage ermuntern, die Identität der Kommunikationswissenschaft zu erkunden. ${ }^{1}$ Es scheint mehrere fachhistorische Anfänge zu geben. Institutionell, personell, theoretisch und methodisch expandiert das Fach im deutschen Sprachraum seit etwa dreißig Jahren graduell unterschiedlich: als empirische Projektforschung und überwiegend als Journalistiklehre. Für die Journalistik kamen gewichtige Anstöße - und Ressourcen - von außen. $\ 2$ Hochschulrahmengesetz (1976) trägt den Hochschulen auf, durch Anwendung wissenschaftlicher Erkenntnisse und Methoden auf künftige Berufstätigkeiten vorzubereiten. Das weltweit verbreitete Hochschulfach Kommunikationswissenschaft [Communications oder Communication Science] firmiert im deutschen Sprachraum opportunistisch: vormals als Zeitungswissenschaft, heute häufig in Verbindungen mit Publizistikwissenschaft, Medienwissenschaft und/oder Journalistik. Dafür standen hierzulande bis in die 1970er Jahre eine Hand voll Professoren mit nicht viel mehr Assistenten und Hilfskräften zur Verfügung. Gleichwohl hielt die Hochschulpolitik das Fach

1 Dem Aufsatz liegt der Text eines Vortrags zugrunde, der anlässlich der Festveranstaltung „20 Jahre Kommunikationswissenschaft an der Otto-Friedrich-Universität Bamberg “ am 20. Juni 2003 referiert wurde - in Reflexion auf meine Bamberger Antrittsvorlesung Kommunikationswissenschaft zwischen Wunsch und Machbarkeit (Rühl, 1985). 
für zuständig und geeignet, berufstaugliches Journalismuswissen selbstkritisch zu lehren.

Den Start vollzogen die LMU München (1973), die Universitäten in Dortmund und Hohenheim (1976), nach zehn Jahren kam die Kommunikationswissenschaft als Journalistik an die Universitäten Bamberg und Eichstätt. Wenige blicken einhundert Jahre zurück, als die Universität Zürich Oscar Wettstein für Journalistik habilitierte. Er war ein Schüler Adolf Kochs, der sich seit 1895 bemühte, der Journalistik an der Universität Heidelberg durch Vorlesungen und praktische Übungen Universitätsreife zu verleihen (Wettstein, 1907; Obst, 1986).

Eine längere Tradition hat die Hypothese, dass das Fach 1915/16 mit der Institutionalisierung der Zeitungskunde an der Universität Leipzig durch Karl Bücher beginnt. Erweitert man die Suche nach Anfängen über die universitäre Institutionalisierung hinaus, dann sind die klassische Rhetorik und Sophistik (Arnold \& Bowers, 1984; Baumhauer, 1986) nicht zu übersehen. Man kann aber auch die Forschungs- und Lehrleistungen des Frühaufklärers Christian Thomasius ins Kalkül ziehen, nicht zuletzt die Zeitungslesepolitik Kaspar Stielers am Ende des 17. Jahrhunderts (Rühl, 1999).

In Deutschland gibt es gegenwärtig rund 50 Hochschulstandorte, an denen kommunikationswissenschaftlich geforscht und gelehrt wird. Einen Mangel an studentischer Nachfrage hat das Fach meines Wissens nie gekannt. Seit den 1990er Jahren verschieben sich allerdings die Zahlenverhältnisse zwischen Lehrenden und Lernenden dramatisch, mit Blick auf die Qualität des Faches durchaus bedrohlich. Aus Budgetgründen werden kommunikationswissenschaftliche Studiengänge „verschlankt“ oder „weggespart“. In der Forschung dominiert nach wie vor die empirische Projektforschung, während ein Interesse an kommunikationswissenschaftlicher Grundlagenforschung erst zögernd belebt wird (Löffelholz \& Quandt, 2003; Ruhrmann et al., 2000; Saxer, 1999; Bonfadelli \& Rathgeb, 1997). Bemühungen um eine Allgemeine Kommunikationswissenschaft [General Communications] sind dagegen unbekannt, auch in Amerika. Ihr Fehlen hat zur Folge, dass zahllose Ergebnisse aus empirischen Forschungsprojekten in alle Richtungen davonfluten. Sie können nicht zu kommunikationswissenschaftlichen Erkenntnissen werden, weil ihnen der innere Zusammenhalt fehlt.

\section{Wiedereintreten in Kommunikation/Gesellschafts-Verhältnisse}

In der Absicht, an der Theorie einer Allgemeinen Kommunikationswissenschaft zu arbeiten, sind zunächst einige wichtige Problembereiche anzuleuchten. Das Unternehmen steht unter der Leitthese: Jede Kommunikationssemantik variiert mit weltgesellschaftlichen Emergenzen. Im Europa des Buchdrucks, der Aufklärung und der Industrialisierung verändert sich die allgemeine Kommunikation, insbesondere die öffentliche Kommunikation, das Publizieren, besonders nachhaltig (Rühl, 1999). Ende des 17. Jahrhunderts unterscheidet und koordiniert Christian Thomasius die Begriffe Mensch, Gesellschaft und Kommunikation. Er postuliert: Der Mensch wird durch die Gesellschaft zum Einzelnen, weil communiciren mit anderen Menschen die Gesellschaft voraussetzt (Thomasius, 1692/1995: 89). Dieser „ärgerliche Neuerer“ (Ernst Bloch) hält 1687 an der von der protestantischen Scholastik dominierten Universität Leipzig die erste Vorlesung in deutscher Sprache (nicht mehr Lateinisch), in eleganter Kleidung (nicht mehr im Talar), über die Schrift Hand-Orakel und Kunst der Weltklugheit des spanischen Jesuiten Baltasar Gracián. Thomasius bekämpft Folter und Hexenverbrennungen, gründet 1688 die deutschsprachige „gelehrte Zeitschrift“ Monats-Gespräche (Kurztitel), geschrieben von Gelehrten, aber nicht nur für Gelehrte. Die Monats-Gespräche unterstützen die 
„Zeitungskollegien“, die Thomasius an der neuen brandenburgisch-preußischen Universität Halle abhält, als er von der kursächsischen Universität Leipzig mit Vorlesungsund Schreibverbot belegt wird. Die Freiheit macht Thomasius für jeden Fortschritt verantwortlich: „Es ist ungebundene Freyheit [...] die allem Geiste das rechte Leben giebet, und ohne welche der menschliche Verstand, er möge sonsten noch so viel Vortheil haben [...] gleichsam todt und entseelt zu seyn scheinet." (Thomasius, 1692/1994: 458-459).

Mensch, Kommunikation und Gesellschaft als aufeinander bezogene Dreiheit zu begreifen, war neu und wird erst wieder am Ende des 19. Jahrhunderts von Emile Durkheim aufgegriffen. Er, Max Weber, Georg Simmel, dann Talcott Parsons erkennen im Akteurshandeln [social action] die Grundlage der Sozialprozesse Arbeitsteilung und soziale Differenzierung. Durkheim und Parsons behaupten, dass nicht Subjekte die Gesellschaft, sondern die Gesellschaft Subjekte hervorbringt (Luhmann, 1977). Max Weber definiert soziales Handeln als sinnverbundene Bezugnahme auf das Verhalten anderer (Weber, 1922/1985: 1). Talcott Parsons kennzeichnet sein eigenes Werk rückblickend als das eines Soziologen und Theoretikers der allgemeinen Handlungstheorie (Parsons, 1973: 33). Und Niklas Luhmann entwirft in unseren Tagen eine Theorie der Weltgesellschaft als Kommunikationssystem (Luhmann, 1997).

Dieser wissenschaftshistorische Aufriss für die Grundlegung einer Allgemeinen Kommunikationswissenschaft muss hinsichtlich sachlicher, sozialer und zeitlicher Dimensionen vertieft und erweitert werden. Interessiert sich die Rezeptionsforschung heute so gut wie ausschließlich für das Bewirken und Nutzen „neuer Medien“, dann sorgen sich, so scheint es, nur Journalisten und Verleger um das Zeitungslesen. Dies verwundert einigermaßen, werden doch Zeitungen seit 400 Jahren in unterschiedlichen Gesellschaftsmilieus gelesen und vorgelesen (Rühl, 2002). Kaspar Stielers Zeitungs Lust und Nutz (1695) ist eine gesellschaftstheoretische Zeitungslesepolitik. Stielers lesepolitisches Plädoyer „von der Zeitungen Notwendigkeit“ war an unterschiedliche Gruppierungen der absolutistischen Schichtengesellschaft Europas gerichtet, namentlich an Kaufleute, Höfe, Kirche, hohe Schulen, Frauen-Zimmer, Reisende, an solche „bey Trunke und Zusammenkunften“, und an die „bey der Statskunde Beflissenen insonderheit“. Die „ehrlichen Leute“ sollten durch Zeitungslesen den frühbürgerlichen Alltag besser verstehen (Stieler, 1695/1969: 4-6, 112-121; Rühl, 1999: 91-102). Funktional vergleichbare Absichten verfolgte die UNESCO nach dem Zweiten Weltkrieg mit ihrer Kampagne für eine „funktionale Literalität“ („functional literacy“), mit der die Bevölkerung postkolonialer Gesellschaften literalisiert werden sollte (Harman, 1970; 1987).

Wird versucht, empirische Beziehungen und Unterschiede zwischen Journalismus, Werbung, Public Relations und Propaganda als Formen der Persuasion und der Manipulation auszumachen (Rühl, 2001; 2003), dann gilt es dafür Funktionen, Leistungen und Aufgabenstellungen herauszuarbeiten. Mit solchen Differenzierungsproblemen war der Arzt Théophraste Renaudot noch nicht befasst, als er im frühen 17. Jahrhundert im 400000 Einwohner zählenden Paris mit einem Elendskreislauf konfrontiert war, bestehend aus Armut, Unterernährung, Krankheit, fehlender Bildung und Dauerarbeitslosigkeit. Protegiert vom Ersten Minister Kardinal Richelieu, dem Theoretiker und Politiker des Absolutismus, entwirft Renaudot eine Woblfabrtspolitik des organisierten Helfens, die im Merkantilismus, der Wirtschaftstheorie des Absolutismus, nicht vorgesehen war. Renaudots Unternehmen bestand aus einer sozialökonomischen und publizistischen Leistungskoordination auf drei Ebenen. Er gründet und betreibt (1) das Bureau d'Adresse et de rencontre, eine Mehrzweckorganisation des Tauschens, Vermittelns, Beratens, Diagnostizierens und Therapierens, (2) das Feuille $d u$ Bureau 
d'Adresse, eine Angebots- und Nachfrageliste, in der gebrauchte, verlorene und gestohlene Sachen, Lotteriegewinne, Geburten, Hochzeiten und Trauerfälle angezeigt und Dienstboten, Reisebegleiter, Kapitalien und Grundstücke nachgefragt werden, und (3) teils persuadierende, teils manipulierende Zeitungen, mit der Gazette als dem Leitorgan (Solomon, 1972; Rühl, 1999: 83-90).

In der Mitte des 18. Jahrhunderts beobachtet der damals Moralphilosophie lehrende Adam Smith die Kommunikationsqualitäten der Selbst- und Fremdbeobachtung. Sie sollen den Menschen kraft ihres Vorstellungsvermögens [imagination] und Mitfühlenkönnens [fellow feeling; sympathy] möglich sein (Smith, 1759/1976: 110). In Smiths Theorie der Wirtschaftsgesellschaft [commercial society] kommt die auf eine Vernunftrationalität reduzierte Denkfigur des Homo oeconomicus zwei Mal vor (Smith, 1776/1974). Von Adam Smith ist bekannt, dass seine Bücher und Manuskripte aus der Ethikvorlesung heraus erarbeitet wurden (Rühl, 1999: 103-111). Es gilt, vermeintliche oder tatsächliche Widersprüche in Smiths Menschenbild aufzuhellen, vor allem aber seinen Gesellschaftsentwurf nicht nur unter marktwirtschaftlichen, sondern vor allem unter Kommunikations- und Handlungsgesichtspunkten zu untersuchen.

Zur gleichen Zeit ersinnt der mehrere empirische Disziplinen mitbegründende Politikwissenschaftler August Ludwig Schlözer (1777) das Zeitungslesen als akademisches Lehrmittel. Robert Eduard Prutz (1845/1971) überlegt Journalismus und Demokratie als Einheit in Differenz, und einer der ersten Sozialwissenschaftler, Albert Schäffle (1875-1878), konstruiert aus geschichtlichen Begriffen (System, Kommunikation, Handeln, Organisation, Öffentlichkeit, öffentliche Meinung, Volk, Nation) eine Gesellschaftstheorie mit einer Publizistik (Hardt, 1979; Rühl, 1999).

Seit ihren Anfängen (1892) studiert man im Umfeld der University of Chicago die Humankommunikation als Organisieren und Ordnen (Cooley, 1902/1964; 1909/1962; Mead, 1934/1967). Die Journalistik gab es schon an einigen Colleges, aber sie war noch nicht wissenschaftsfähig [researchable] (Rogers \& Chaffee, 1994). Eine Apologetik der Publizistik, die rechtfertigen sollte, dass „publizistische Persönlichkeiten“ mittels Gesinnungskräften auf die Öffentlichkeit einwirken (Dovifat, 1971: 1-5), blieb auf den deutschsprachigen Raum begrenzt. Nach dem Zweiten Weltkrieg setzt sich im Westen Deutschlands das Fünf-Fragen-Schema Harold D. Lasswells als Lehrmodell durch. Die Lasswell-Formel war 1939/40 in das Rockefeller Communication Seminar als Arbeitshypothese eingebracht worden (Rogers, 1994: 222), und wurde dem Aufsatzklassiker The structure and function of communication in society (Lasswell, 1948/1987) vorangestellt. Seither dient sie vielen Kommunikationsmodellen als Vorbild oder als Gegenbild (McQuail/Windahl, 1998).

Parallel dazu bringt es eine methodologisch in Wien konzipierte empirische Sozialforschung (Jahoda/Lazarsfeld \& Zeisel, 1933/1975) in Amerika zu einer anwendungsorientierten Medienforschung. Sie ist begrifflich-theoretisch auf Kleingruppe [small group], Verhalten [behavior], Meinung [opinion], Einstellung [attitude] und Wirkung [effect] ausgerichtet (Lazarsfeld/Berelson \& Gaudet, 1944/1968; Katz \& Lazarsfeld, 1955). In der Kooperation zwischen den Fakultätskollegen Paul F. Lazarsfeld und Robert K. Merton an der Columbia University werden anfangs der 1940er Jahre Unterschiede zwischen angewandter Forschung und Grundlagenforschung akzentuiert (Lazarsfeld, 1981). Beide tendieren zu unterschiedlichen kommunikationswissenschaftlichen Forschungsrichtungen. Merton bestimmt für die Massenkommunikation einen sozialstrukturellen, teleologisch-funktionalen Forschungstrend (Wright, 1959/1986), während Lazarsfeld psychischen Wirkungen [psychic effects] der Massenmedien nachspürt, die an Motiven, Einstellungen und Meinungen gemessen werden. 
Als sich die von Nationalsozialismus und Zweitem Weltkrieg mäßig erholte deutschsprachige Kommunikationswissenschaft in den 1960er Jahren entschließt, eine Sozialwissenschaft zu werden (Dröge/Lerg, 1965; Prakke et al., 1968; Ronneberger, 1964/1974; 1970), forciert - in der Nachfolge von Lazarsfeld - Elisabeth Noelle-Neumann (1989) eine empirische Publizistik(wirkungs)forschung - ohne höhere sozialtheoretische Ambitionen. Soziale Auswirkungen [social impacts] sind dieser Forschungsrichtung ziemlich fremd (Schulz, 1986: 6). Wird beispielsweise beabsichtigt, den britischen und den deutschen Journalismus zu vergleichen (Esser, 2000; Köcher, 1985), dann richtet sich das Interesse nicht auf Journalismussysteme als Sozialitäten, sondern auf psychische Befindlichkeiten von Befragungskollektiven. Dieser Wirkungsforschung geraten Gesellschaft und Geschichte aus dem Blick, vor allem der Kommunikationsbegriff, so dass von ihren Vertretern nicht gesagt werden kann: „(Re)conceptualizing communication, talking or writing of communication, that is, communication of communication, is what we communication scholars do" (Krippendorff, 1996: 311).

\section{Kommunikationswissenschaftliches Erkennen}

Bevor wir auf normaltheoretische Probleme der Kommunikationswissenschaft eingehen, sind erkenntnistheoretische und methodentheoretische Zugangsweisen in wenigen groben Strichen zu skizzieren. Mit Epistemologie und Methodologie versuchen Wissenschaftler anderen Wissenschaftlern zu erklären, was sie (oder die anderen) getan haben oder tun können. Es kommt keine brauchbare Wissenschaft heraus, schreibt man Wissenschaftlern vor, was sie zu tun haben. Selten wird fachintern diskutiert, welche Erkenntnis- und Methodentheorien der Kommunikationswissenschaft zur Verfügung stehen (Anderson, 1996).

In ihrer zeitungswissenschaftlichen Phase wählt die Kommunikationswissenschaft eine erkenntnistheoretische Idealvorstellung von Zeitung, die sie mit der methodentheoretischen Betrachtungsweise der Historik untersucht. Zusammen mit zeitlich unabhängigen, empirisch unzugänglichen Wesensmerkmalen (Periodizität, Universalität, Aktualität, Publizität), entlehnt sie der Scholastik die Komplementärbegriffe Idealobjekt und Materialobjekt. Idealobjekten wird ein Auswahlpotenzial zugeschrieben, in das Materialobjekte, die als Datenmaterial gegeben sind, hineinverlagert werden (Groth, 1960: Bd. 1; 1913/1915). Für die daran anschließende Publizistikwissenschaft ist, zusammen mit Zeitung, Zeitschrift, Rundfunk und Film, die Publizistik als Realität faktisch da, und aus diesem Publizistikverständnis werden Normativitäten (Gesinnung, Moral, Richtigkeit) abgeleitet. Diese Theorientradition wird hier nicht weiter verfolgt. Stattdessen soll versucht werden, die seit einem Jahrzehnt (Bentele \& Rühl, 1993; Bonfadelli \& Rathgeb, 1997) im deutschen Sprachraum widerstreitenden Strömungen des Realismus und des Konstruktivismus in Grundzügen zu typisieren.

Realisten operieren als Kommunikationswissenschaftler üblicherweise mit einer Subjekt/Objekt-Rationalität. Kommunikation, Medien oder Journalismus sind für sie alltagsvernünftig verstehbare Gegebenheiten. Journalisten existieren real, der Journalismus besteht aus realen Gegenständen, Subjekten und Produkten (Kepplinger, 2000; Pöttker, 2000). Diesen ontologischen Einschätzungen würde kein Neopositivist und kein Neomarxist widersprechen können. Realisten definieren anschaulich, und sie halten Definitionen selbst für Wirklichkeiten, deren Trefflichkeit und Richtigkeit durch Aussagen „großer Praktiker“ bestätigt werden können.

Nun gilt für die Wissenschaft grundsätzlich, dass sie ihre Forschungsprobleme selbst aufstellt und formuliert, diese mit eigenen Methoden bearbeitet, die Arbeit selbst kon- 
trolliert, in der Regel vor dem theoriegeschichtlichen Horizont eigener Wissensbestände. Auch Kommunikationspraktiker bedenken die von ihnen formulierten Kommunikationsprobleme epistemisch und methodisch, allerdings anhand „praktischer" Sichten (Rühl, 2000a; ausführlicher: Rühl, 2004a). Wenn kommunikationswissenschaftliche Realisten in ihren Lehrbüchern wissenschaftliche Aussagen und die Aussagen von Praktikern „gleichberechtigt“ nebeneinander stellen, dann können sie nicht erwarten, dass beide Aussagetypen über vergleichbare Erklärungskraft verfügen.

Anhand der klassischen Methodologie werden von Realisten die Forscher als gestandene Subjekte konzipiert, die Weltprobleme projektförmig vereinfachen und durch quantitative und qualitative Forschungsmethoden „richtig“ beschreiben können. Es mangelt an einer reflektierten Distanz zu vorwissenschaftlichen Realitätsverständnissen sowie an der Selbsterkenntnis, dass jede wissenschaftliche Lehre und Forschung jedes Problem gleichzeitig beeinflusst. Von Realisten wird die Hyperkomplexität der Welt auf wenige, in sich abgeschlossene Variable reduziert. Es werden Beziehungen, Korrelationen und Abhängigkeitsbedingungen zwischen Variablen hergestellt, um den „Rest der Welt" der Ceteris paribus-Formel zu überantworten, die besagt, dass alles andere beim Alten bleibt.

Leugnen Realisten beispielsweise die Möglichkeit einer wissenschaftlichen Theorie des Journalismus (Kepplinger, 2000: 81), dann belegen sie, dass sie eine Theorie über eine (angebliche) Nicht-Theorie formulieren. Methodentheoretisch bevorzugen Realisten die Empirik - nicht die Phänomenologie, den Funktionalismus, die Dialektik oder die Historik - und seit einigen Jahrzehnten scheint es so zu sein, als ließen sich alle kommunikationswissenschaftlichen Fragen mithilfe der empirischen Verfahren „Befragung“ und „Inhaltsanalyse“ beantworten. Werden bestimmte Journalisten als ausgewählte Kollektive über milieubedingte Erlebnisse befragt und werden aus ihren Antworten statistische Schlüsse gezogen, dann ist zu fragen, welche kommunikationstheoretischen Evidenzen aus statistisch bearbeiteten Daten zu erwarten sind? Werden "gegebene“ Texte, Inhalte, Abbildungen, Musik- und Theateraufführungen als manifest gekennzeichnet und als ontisch objektivierbare Zeichenkombinationen gedeutet (Berelson, 1952; Merten, 1995), dann interessiert: Wie kommen die Zeichen zu dem Sinn, den die Forscher aus ihnen herauslesen? Noch immer ist Abraham Kaplans Verdacht nicht ausgeräumt, der sein (ironisches) „law of the instrument“ wie folgt erläutert: „It comes as no particular surprise to discover that a scientist formulates problems in a way which requires for their solution just those techniques in which he himself is especially skilled“ (Kaplan 1964, 28). Verzichten realistisch operierende Kommunikationswissenschaftler ausdrücklich auf Theoriearbeit, dann interpretieren sie ihr Datenmaterial dennoch theoretisch, nämlich laientheoretisch, durch jedermanns Lebenserfahrungen.

Konstruktivistisch operierende Kommunikationswissenschaftler widersprechen den Realisten auf mehreren Ebenen. Für Konstruktivisten sind Gegebenheiten alltagsvernünftige, das heißt nicht wissenschaftsfähige Mutmaßungen. Konstruktivisten fragen grundsätzlicher: Wie kann man kommunikationswissenschaftliche Probleme erkennen und ordnen, obwobl deren empirische Erkenntnis keinen Zugang zu einer Realität hat außerhalb ihrer selbst? Bedienen sich Konstruktivisten der funktional-vergleichenden Methode, dann distanzieren sie sich dadurch von den teleologischen und den mechanischen Kausalitäten, wenn sie Kommunikationsprobleme system(mitwelt)rational problematisieren. Kommunikation wird zum Schlüsselbegriff. Kommunikation lässt sich in die Komponenten Information, Sinn, Thema, Äußerung, Erinnern und Verstehen aufbrechen, auswählen, abwandeln und rekombinieren. Anders als Verhalten und Handeln verfügt Kommunikation über eine hochkomplexe, konkurrenzlose semantische Öff- 
nungskraft. Mit Kommunikation können Menschen irritieren, ironisieren, manipulieren, kurz: widersprüchlich, ja widersinnig kommunizieren. Ein kommunikativer Perfektionsanspruch wie die Habermas'sche Verständigung kann kein „konstantes Ziel“ (Burkart, 1998: 26) der Kommunikation sein. Kommunikation reproduziert sich nach konstruktivistischer Auffassung selbst, und zwar dreidimensional: in der sachlichen, der sozialen und in der zeitlichen Dimension. Wir kommen darauf zurück.

Wir verstehen uns recht: Realismus und Konstruktivismus können nicht die einzig richtigen (oder falschen) Methoden sein, für (oder gegen) die man sich als Kommunikationswissenschaftler entscheiden muss, um die getroffene Wahl mit Bekennermut zu missionieren. Realismus und Konstruktivismus stehen in der Kommunikationswissenschaft methodisch mit Phänomenologie, Funktionalismus, Dialektik und Historik im Wettbewerb. Doch zuerst sind Forschungsprobleme zu formulieren, sie haben den Forschungsprimat. „Das empirische Denken ist klar erst im nachhinein, wenn der Apparat der Erklärung zum Zuge gekommen ist" (Bachelard, 1938/1978: 46). Erst dann kann entschieden werden, von welchen Methoden bzw. von welchen methodischen Verfahren man sich für eine Lösung der Probleme die größeren Erfolgschancen verspricht.

\section{Was hat Medienforschung mit Kommunikationsforschung zu tun?}

Für die Alltagsvernunft ist Kommunikation „ein gutes Wort“. Dagegen kommt der Medienbegriff irrlichternd daher (Rühl, 1998). Zunächst nannte man Presse, Radio, Film und Fernsehen „die Massenmedien“. Bald kam der Wortteil „Masse“ abhanden, und unter dem Begriffstitel „Medien“ werden seither Videos, Handys, Computer und manch andere Techniken bzw. Technologien versammelt (Rühl, 2000b).

Seit der handlungsnahen Philosophie des Aristoteles gehören Medien bzw. Mittel zum festen Bestand abendländischer Lehrtraditionen. Aristoteles (1979: 1111b) konzipiert Mittel für den Zweck, zu dem sie gebraucht werden. Zwecke werden von ihm final gedacht, als gewünschte Ergebnisse eines anzustrebenden Guten, das durch dinghafte Mittel erfüllt werden kann. Medien werden mit der Teleologie, das ist die Lehre von den Zweck/Mittel-Verhältnissen, realbegrifflich als „the nature of“ beschrieben, in Gattungen, Arten und Unterarten gegliedert und durch wesentliche oder unwesentliche Eigenschaften und Merkmale qualifiziert. Ihre begriffstheoretische Vorgeschichte verdient eine genauere Analyse, die an dieser Stelle nicht zu leisten ist (Rühl, 2000c). Stattdessen werfen wir wenige Schlaglichter auf den Medien- bzw. Mittelbegriff in seiner kommunikationswissenschaftlichen Karriere.

In der Mitte des 19. Jahrhunderts bezeichnet Karl Knies die Eisenbahn und den Telegraphen als Communicationsmittel (Knies, 1853; 1857), die er - nach heutigem Sprachgebrauch - sozialökonomisch als Transportmittel für Güter, Nachrichten und Menschen untersucht (Rühl, 2003). In der Mitte des 20. Jahrhunderts bemüht sich der Ingenieur Claude E. Shannon (Bell Telephone Laboratories) um preiswerte Transporte von Signalen (Shannon/Weaver, 1949). Ihn beschäftigen kostengünstige Einweg-Transporte, und Shannon formuliert Theoreme über Kanalkapazität und Informationsmessungen und nennt das Ergebnis seiner Arbeit „mathematical theory of communication“. Den technisch gemeinten Begriff communication erläutert sein Co-Autor Warren Weaver psychologisch als „the procedures by which one mind may affect another" (Weaver, 1949: 3). Wilbur Schramm, der das Shannon/Weaver-Buch im Verlag der University of Illinois lektoriert hatte, popularisiert anschließend Shannons Theorie unter dem Titel „information theory“. David K. Berlo transformiert die Kernbegriffe Shannons (Quelle, Kanal, Mitteilung, Empfänger, Rauschen, Rückkopplung) aus einem Technolekt in 
einen Soziolekt. Seinem Buch The process of communication (1960) liegt, ungeachtet des Titels, ein Verhaltensmodell für öffentliche Persuasion zugrunde, das in den 1960er und 1970er Jahren das Lehrbuch in der nordamerikanischen Kommunikationswissenschaft wird (Rogers, 1994: 416-417).

Zur gleichen Zeit führen Sozialpsychologen die Begriffe Massenkommunikationsmedien, Massenkommunikation und Medien in die Lehrbuchliteratur ein (Wiebe, 1955; Maletzke, 1963). Dabei konnten sie sich semantisch auf die Frucht tragende Zusammenarbeit zwischen Paul F. Lazarsfeld und Robert K. Merton (Lazarsfeld, 1981) berufen. Lazarsfeld akzentuiert für Massenkommunikation den Mitteilungstransfer via Massenmedien (Zeitung, Zeitschrift, Film, Radio, Fernsehen), während die Merton-Schüler betonen: „Mass communication is organized communication“ (Wright, 1959/1986: 15). Damit wird die sozialwissenschaftliche Organisationsforschung angesprochen, die seit Beginn des 20. Jahrhunderts die Sozialwissenschaften zunächst handlungstheoretisch umbricht (Walter-Busch, 1996; Ortmann et al., 1997) - seit den 1950er Jahren in Amerika zunehmend kommunikationstheoretisch (Theis, 1994/2003; Jablin et al., 1987; Luhmann, 2000). In der kommunikationswissenschaftlichen Forschungspraxis werden Verlag, Anstalt und Agentur durchaus beim Namen genannt, wenn auch selten sozialempirisch problematisiert. Es blieb der deutschsprachigen Redaktionsforschung vorbehalten, organisationsspezifische Erkennungsregeln (Mitgliedschafts-, Arbeits- und Berufsrollen, Stellen und Stellennetze, organisationsinterne Normen und Zeiten, Zuständigkeit und Verantwortung, geordnete Entscheidungsprogramme, Redaktions- und Ressortstrukturen im Wandel) empirisch zu untersuchen (Rühl, 1989/2003; Rühl, 2004b).

"Communication is the process of transmitting meaning between individuals“ (Wright, 1959/1986: 7). Von der Idee, die menschliche Kommunikation als Vermittlung zwischen cartesianischen Cogito-Subjekten [„Ich denke, also bin ich“] zu verwirklichen, kann sich die Kommunikationswissenschaft nur mühsam befreien, zumal dann, wenn sie Kommunikation sagt und Massenkommunikation meint. Drei Hauptrichtungen werden unterschieden: (1) das Transmittieren von Mitteilungsmengen, das (2) Öffentlichkeitscharakter hat und (3) relevant ist für die Massengesellschaft (McQuail, 1989: 488). Auf dieser Denkebene werden dann die teleologischen Funktionen der Massenkommunikation ausdifferenziert (Wright, 1959/1986; Ronneberger, 1971).

Die Medienwirkungsforschung lässt offen, wie die Outputs vortypisierter Vermittlungs-, Übertragungs-, Nachrichten- oder Unterhaltungsmedien semantisch in Beziehung zu setzen sind zu den Formen öffentlicher Kommunikation, zu Informationen, Themen und zur öffentlichen Meinung. Befragungen und Inhaltsanalysen stehen in alten und festen teleologischen Überlieferungen, ohne dass Sinnmachen [sensemaking] (Weick, 1995) zum Kommunikationsproblem wird. Empirisch verfahrende Medienforscher haben in Gestalt von Datenerhebungen, Messverfahren, statistischen Datenanalysen und Experimentiertechniken eine eigene Forschungstechnologie geschaffen. Doch so lange keine inkongruenten Theorieperspektiven gewählt werden, die alternative Kommunikationsprobleme aufzeigen können, werden noch so gewitzt prozessierende Befragungen und Inhaltsanalysen keine wissenschaftlich-hypothetischen Wahrheitserfolge ergeben.

Eine frühe erkenntnis- und methodentheoretische Selbstkritik der empirischen Sozialforschung fand offenkundig kein nachhaltiges Echo. So reflektierte Robert S. Lynd, der zusammen mit Helen M. Lynd die stadtsoziologischen Klassiker Middletown (1929) und Middletown in transition (1937) verantwortete, in Knowledge for what? (1939/1970: 119-120) die eigene empirische Forschungsarbeit: „To carry it on, one usually places oneself inside the going system, accepts temporarily its values and goals, and sets to work 
at gathering data [...] One tends to be drawn deeper within the net of assumptions by which the institution he is studying professes to operate." Methodologische Konsequenzen sind von der empirischen Kommunikationsforschung wohl nicht gezogen worden.

Werden kommunikationswissenschaftliche Probleme mit Luhmanns „Konstruktivismus" konzipiert, dann liegt nahe, die als komplementär verstandene, funktional-vergleichende Methodentheorie heranzuziehen. Werden indes empirisch-neopositivistische Verfahren (Befragung, Inhaltsanalyse, Experiment) gewählt (Loosen/Scholl \& Woelke, 2002: 37-65), dann droht ein Methodenschisma (Rühl, 1986: 86-90). Luhmanns System(mitwelt)theorie erlaubt, Kommunikationssysteme intern zu strukturieren, um damit den widerspruchsvollen Anforderungen der Weltgesellschaft in Autonomie zu begegnen, zur Stabilisierung der Kommunikations/Gesellschafts-Verhältnisse. Empirische Vergleichstechniken der funktional-vergleichenden Methode stehen in der Erkenntnistradition Immanuel Kants (1788/1968: B 93) und Ernst Cassirers (1910/1990). Insofern werden Funktionen nicht als konkrete, Zwecke erfüllende Zielvorstellungen ausgedacht, sondern als abstrakte Gesichtspunkte begriffen, von denen aus präzisierende Bezugsprobleme funktional äquivalent zu behandeln sind. Was Niklas Luhmann (1970) abgeklärte Aufklärung nennt, kann kommunikationswissenschaftlich als das Aufdecken latenter Technisierungen, Medialisierungen und Thematisierungen von Kommunikation beobachtet werden, ohne vollkommene Information und ohne eindeutigen Sinn als Auslöser von Resonanz zu postulieren (Rühl, 1988). Von den genannten empirisch-beschreibenden bzw. empirisch-analytischen Verfahren ist bekannt, dass sie durchaus auf Systeme bezogen werden, die als Aggregate essenzieller Subjekte verstanden werden, womit Kommunikation nicht zu messen ist.

Johann Beckmann, Freund und Kollege Schlözers in Göttingen, erschließt in der zweiten Hälfte des 18. Jahrhunderts eine Allgemeine Technologie, unter anderen am Gegenstand Papier (Beckmann, 1783-1793/2002). Die Kanadier Harold A. Innis und Marshall McLuhan observieren Technologien der Kommunikation, und zwar deterministisch. Während sich Innis (1950/1972; 1951/1982) für technische Kommunikationswirkungen auf Wirtschaftsorganisationen interessiert, wird McLuhan mit einer Mediologie weltberühmt. Er beobachtet vom Standpunkt der ontologischen Metaphysik die menschlichen Sinne, das Hören, Sehen, Fühlen, Riechen und Schmecken. Ohne in seinen Publikationen auf kommunikationswissenschaftliche Forschungen einzugehen, behauptet McLuhan das Seiende der Medien für beständig. Das tat vor ihm schon Wolfgang Riepl (1913/1972) methodisch medienhistorisierend, während McLuhan einem theologisierenden Biologismus anhängt, nach dem Medien nicht nicht sein können. Medien sollen zu fantastischen Expansionen des menschlichen Körpers verhelfen: das Rad zur Erweiterung des Fußes, das Buch zur Erweiterung des Auges, die Kleidung zur Erweiterung der Haut, der Stromkreislauf zur Erweiterung des Zentralnervensystems (McLuhan/Fiore, 1967). „All media, from the phonetic alphabet to the computer, are extensions of man that cause deep and lasting changes in him und transform his environment" (McLuhan, 1969: 58). Marshall McLuhans Medienideen sind von der empirisch kontrollierenden Kommunikationsforschung nie geprüft worden.

Eine aus der Literaturwissenschaft emanzipierte Medienwissenschaft (Rusch, 2002) beansprucht, die Medienwissenschaft zu sein - ohne vergleichende Medienuntersuchungen anzustellen. An sie sind grundsätzliche Fragen zu richten: Ist die Medienwissenschaft eine Disziplin, eine Interdisziplin, eine Ko-Disziplin, mit welchen Begründungen? Sind Medientheorien Technologietheorien oder Bewusstseinstheorien, Begleittheorien oder Supertheorien? Taugen die wissenschaftlichen Leistungen der Me- 
dienwissenschaft, um die Kommunikationswissenschaft hochschul- und förderungspolitisch zu agitieren? Wird es wissenschaftlich fruchtbarer, wenn beide - Medienwissenschaft und Kommunikationswissenschaft - komische Bettgenossen [odd bedfellows] genannt oder als „Nachbardisziplinen“ begriffen werden, unter dem Slogan: Every week is good-neighborhood-week?

Es ist bisher nicht gelungen, für Medien und Kommunikation eine gemeinsame Reflexionsebene und praktikable Forschungsmethoden zu finden, die erlauben, Medien in Kommunikation zu transformieren (Löffelholz, 2003). Greift man Luhmanns (1992: 181) Fragenkombination auf: „Was ist ein Medium? Was ist ein Kommunikationsmedium? Was ist ein symbolisch generalisiertes Kommunikationsmedium?", dann sind in erster Linie epistemische und methodische Medien- und Kommunikationsfragen zu diskutieren. Das „Selbstverständnispapier“ der Deutschen Gesellschaft für Publizistikund Kommunikationswissenschaft (2001) beschreibt viel Medienforschung aus unterschiedlichen Sichten, weniger Forschungen, die um Kommunikation rotieren. Mit dem Papier will man nach draußen wirken, will für die Kommunikationswissenschaft Public Relations betreiben. Grundlagentheoretische Ansprüche formuliert das Papier folglich keine.

\section{Erkenntnishindernisse der Kommunikationswissenschaft}

Nach Gaston Bachelard (1938/1978) können wissenschaftliche Erkenntnisse erst gelingen, wenn man Erkenntnishindernisse angeht. Dazu gehört in erster Linie, dass sich die Wissenschaft von nicht-wissenschaftsfähigen Alltagserfahrungen und Erlebnissen distanziert. Wissenschaftliche Erkenntnishindernisse [obstacles épistémologiques] sind hausgemacht. Nicht gemeint sind politische und wirtschaftliche Störungen von außen, auch keine psychischen Leistungsschwächen oder persönliche Vorteilsnahmen von Forschern, die wissenschaftliches Forschen durchaus behindern können. Wissenschaftliches Wissen wird nicht einfach angehäuft. Erkenntnisse werden gegen früheres Wissen gebildet. Empirisches und theoretisches Forschungszeug, das im historischen Verlauf Instrumentencharakter erwirbt, kann den Erkenntnisfortschritt verbiegen, wenn nicht unbrauchbar machen (Bachelard, 1938/1978: 46-58). Daher die Empfehlung, mit Erkenntnishindernissen aufzuräumen [mop-up work] (Kuhn, 1973: 45), wir würden anregen: Erneuerung durch Umbau.

Für die Kommunikationswissenschaft ist der landläufige Kommunikationsbegriff ein besonderes epistemisches Erkenntnishindernis. Ungenau, ungeklärt und unkontrolliert versteckt der in der Kommunikationswissenschaft angewandte Kommunikationsbegriff technische, neurale, animalische, mediale Übertragungen oder Vermittlungen von Etwassen (Impulse, Stimuli, Signale, Zeichen). Dabei verfügt die Menschheit mit der (sprachlichen) Kommunikation über ein unvergleichlich raffinertes Äußerungsvermögen. Sachlich kann Kommunikation sinnmachend informieren, thematisch kann es eingrenzen und zur Weiterkommunikation anregen. Sozial wird Kommunikation in der Familie anders vollzogen als im Büro, im Stadion füllenden Wahlkampf anders als beim Vorlesen von Kinderbüchern im Bett. Bei der Kommunikation der Fernseh-Gameshow oder beim Umbrechen kurz vor Redaktionsschluss entstehen andere Zeitprobleme für das Kommunikationsstudium als beim Beten in buddhistischen Tempeln oder beim Tiere Beobachten in der freien Wildbahn.

Es gibt weder Urzellen der Kommunikation, noch Kommunikation an sich. Dialoge, Diskurse, Gespräche oder Konversationen sind sozial rekonstruierte Kommunikationssysteme, die mit einer kommunikativen Vergangenheit umgehen. Entscheidungs- 
freie Subjekte, Individuen, Akteure, das philosophische Konstrukt „ganzer Mensch“ (Rössler, 1980) können vielerlei, nur nicht kommunizieren. Robinson verfügt über ein Bewusstseinssystem und ein Organismussystem, aber zur Kommunikation braucht er Freitag, besser gesagt: Voraussetzungen und Bedingungen für Kommunikation, die über das eigene Bewusstsein und über die eigene Körperlichkeit hinausreichen.

Erkenntnishinderlich kann der Begriff Pressefreibeit werden, wenn er als das publizistische Grundmuster akzeptierter Werte und Normen in Opposition zu Verbot und Zensur definiert wird. Diese Kennzeichnung ist nicht falsch, aber unzulänglich, da gesellschaftliche Bindungen für Eigen-Sinn fehlen. Der patriarchalisch-absolutistische Obrigkeitsstaat ist die Vorbedingung, dass Pressefreiheit als Fürsorge für Untertanen (Löffler, 1837) gedeutet werden kann. In der Verfassung der Vereinigten Staaten von Amerika (1787) gebietet der Erste Zusatzartikel (1791) dem Parlament: „Congress shall make no law abridging [...] the freedom of speech or the press“. Nach Artikel 5 Absatz 1 Grundgesetz der Bundesrepublik Deutschland gehört die Pressefreiheit zu den Sonderformen der in diesem Lande möglichen allgemeinen Kommunikationsfreiheit (Luhmann, 1965: Kap. 5). Und der sowjetsozialistisch verfasste Staat der Arbeiter und Bauern interpretierte - mit Artikel 1 und 27 der DDR-Verfassung von 1974 - die Pressefreiheit als Verpflichtung gegenüber der marxistisch-leninistischen Einheitspartei.

\section{Erkenntnisfortschritte durch inkongruente Perspektiven}

Erkenntnishindernisse und Erkenntnisfortschritte sind dreidimensional zu lesen. In der Sachdimension der Kommunikationssysteme sind es die Komponenten Sinn, Thema, Information, Mitteilung, Gedächtnis und Verstehen, die, aufeinander bezogen, bewirken können, dass Kommunikation zustande kommt. Dabei steht Sinn für „das Gemeinte“, „das Gewusste“, Information meint „das Neue“, „das Überraschende“, Thema operiert als „das Eingrenzende“, Äußerung (Mitteilung) steht für das „Geäußerte“. Kommunikation erinnert durch psychische Gedächtnisse und soziale Gedächtnisse (Archive, Bibliotheken, Museen), um zu vergegenwärtigen. Verstehen ist „das Gelingen“ des Zusammenspiels der genannten Kommunikationskomponenten. Ausdrücken kann man Kommunikation verbal und nonverbal, durch symbolisierte Kommunikationsmodi (Wort, Gebärde, Bild, Musik), die aus den Gedächtnissen verfügbar gemacht werden (Retention), zum Auswählen (Selektion), Abwandeln (Variation) und zum Umbau (Rekonstruktion) produzierter Kommunikation. Den Dreh- und Angelpunkt der Kommunikation bildet die orale und literale Sprache, mit der stets „Ja“ oder „Nein“ gesagt werden kann (Burke, 1966). Im Modus Sprache der menschlichen Kommunikation ist ein reflexiver Selbstbezug eingebaut, der ermöglicht, mit der Sprache sowohl über die Sprache und über die Kommunikation zu kommunizieren (Luhmann, 1995).

Damit Kommunikation nicht bei jedem Widerspruch oder jeder Zustimmungsverweigerung aufgegeben oder angepasst werden muss, entwickelt sie in der Sozialdimension Systemstrukturen, an denen sie sich orientiert. Funktional-strukturell können allgemeine Kommunikationssysteme (Politik, Wirtschaft, Wissenschaft, Recht, Religion, Publizistik) und spezielle Kommunikationssysteme (Buchpublizistik, Alltagspublizistik, Public Relations, Journalismus, Werbung) unterschieden werden. Für die moderne Weltgesellschaft sind dreierlei Kommunikationssysteme typisch geworden: einfache Kommunikationssysteme (Konversationen, Telefonate, Freundschaften, Skatrunden), organisatorische Kommunikationssysteme (Unternehmen, Parteien, Verbände, Krankenhäuser, Spielbanken) und die genannten gesellschaftlichen Funktionssysteme (Familie, Ein-Personen-Haushalt, Politik, Wirtschaft, Recht, Wissenschaft usw.). Mit ihren 
Sozialstrukturen (Rollen, Stellen, Normen, Werte) halten Kommunikationssysteme Erwartungsstrukturen fest, mit denen die Menschheit zirkulär kommuniziert - in der Erwartung bestimmter und bestimmbarer Mitweltereignisse, auf die sie reagieren und re-reagieren kann (Luhmann, 1984; Rühl, 1987).

In der Zeitdimension ist für die Kommunikation weder ein feststellbarer Anfang noch ein absehbares Ende zu beobachten. Als Kreislauf oder als Spiralverlauf modelliert, wird Kommunikation durch ständiges Wiedereintreten [re-entry] in bewahrte Kommunikationssysteme reproduziert, in Orientierung an den Horizonten der „ererbten“ Kommunikationskulturen der Weltgesellschaft (Rühl, 2001). Zeitdimensional kann die Kommunikationswissenschaft nach konstanten und variablen Kommunikationsfaktoren unterscheiden, nach mündlichen und schriftlichen, verbalen und nonverbalen, archaischen, segmentierten und differenzierten Kommunikationszusammenhängen.

Kommunikationswissenschaftler sind keine Schöpfernaturen. Vielmehr formulieren, bearbeiten und lösen sie Lehr- und Forschungsprobleme hypothetisch, also vorläufig, indem sie mit bewahrten, kommunikationswissenschaftlich relevanten Theorien umgehen. Mit der Erkenntnistechnik inkongruenter Perspektiven [perspectives by incongruity] (Burke, 1935/1965: 69-189) ist Sinn zu erschließen, der kommunikationswissenschaftlich relevant ist, ohne zunächst gemeint zu sein. Dazu einige wissenschaftshistorische und gegenwartsnahe Beispiele.

Karl Marx entwirft - eingebettet in die von ihm als kapitalistisch gedeuteten Gesellschaftsstrukturen des sich gerade industrialisierenden Mitteleuropas - eine Sozialtheorie, den Historischen Materialismus, und zwar anhand kultureller Ideale, die er auf Möglichkeiten wirtschaftlicher Bedürfnisbefriedigung zurückführt. Im fin de siècle Wiens interpretiert Sigmund Freud Träume als unbewusste Wünsche der Alltagskommunikation. Witze, fasziale Ticks, Versprecher, Zwänge, das Tagträumen interpretiert Freud in seiner „Sprache des Unbewussten“. Schon immer greifen Historiker auf Texte über Weltereignisse, Personen und Institutionen zurück, die zu unterschiedlich fernen Zeiten symboldifferent ausgedrückt und von den Historikern in eine Gegenwartssprache übertragen werden. Robert E. Prutz (1845/1971) konzipiert Journalismus und Demokratie als zwei aufeinander bezogene Seiten eines Entwicklungsproduktes (Rühl, 1999: 162-167).

Auch in der modernen Kommunikationswissenschaft werden für alte Sichtweisen inkongruente Perspektiven gewählt. Harold D. Lasswell (1948/1987) schlägt für die Gesamtheit Massenkommunikation drei gesellschaftliche Funktionen vor: das Transparentmachen von Informationen aus der gesellschaftlichen Umwelt, die Interpretation dieser Informationen für die Gesellschaft und die Transmission sozialer Werte und Normen von Generation zu Generation. Robert K. Merton (1957: 19-84) greift Sigmund Freuds Unterscheidung manifester (beabsichtigter) und latenter (unbeabsichtigter) Funktionen auf, wenn er nach den Konsequenzen zweckhaft persuadierender Aktivitäten der Massenkommunikation fragt. Franz Ronneberger (1971; 1978-1986) differenziert die Massenkommunikation als Kommunikationsform zur Sozialisation in der Moderne, formuliert soziale und politische Funktionen, und entwirft eine Theorie der Kommunikationspolitik für die „Wissenschaftlich-Technische Zivilisation“. Manfred Rühl (1969/1979; 1980) hält eine universalistisch orientierte Journalismustheorie für möglich, die er - am sozialwissenschaftlichen Theorienpluralismus orientiert - durch „Schlüsseltheorien“ (nach Luhmann, 1984: 19: „Supertheorien“) funktional vergleichend rekombiniert. Dazu gehören Theorien über System/Mitwelt-Beziehungen, Weltgesellschaft, Kommunikation, Entscheidungen, Organisationen, Märkte, Öffentlichkeit(en), Evolution(en). Der Journalismus kann mikroanalytisch als organisierte soziale Systeme, 
mesoanalytisch als wettbewerbstheoretische Marktverhältnisse und makroanalytisch als Journalismussystem der Weltgesellschaft (mit Beziehungen zwischen Funktionssystemen) unterschieden werden. Überwiegend produktionstheoretische Journalismusperspektiven werden seit den 1990er Jahren, vorwiegend im deutschen Sprachraum, mehr oder weniger inkongruent beobachtet (Löffelholz, 2000; Scholl \& Weischenberg, 1998), während inkongruente Perspektiven einer wettbewerbstheoretischen oder rezeptionstheoretischen Journalismusforschung unbekannt blieben.

Ein inkongruentes Gegenstück in der Sicht auf die Rezeptionsforschung könnte die vorwiegend im englischsprachigen Raum zu beobachtende Literalitätsforschung werden. Das Lesen studiert man im deutschen Sprachraum als Kulturtechnik, primär ausgerichtet auf die Förderung des Buchlesens (Bonfadelli \& Bucher, 2002). Die unter dem Gesichtspunkt der funktionalen Literalität [functional literacy] praktizierte Leseförderungspolitik der UNESCO löste weitere Forschungsvarianten aus, nicht zuletzt das Studium wissenschaftlicher (Il-)Literalität [scientific (il)literacy] (Daedalus, 1983). Kaspar Stielers Zeitungslesepolitik war eine Klugheitslehre für den Alltag in der absolutistischen Schichtengesellschaft Europas, weil „Alexander/Caesar/und Mahomet“ keine Alltagsklugheit lehren würden. Der heutigen Zeitungsleseförderungspolitik - deren Ausmaß kaum der Rede wert ist - fehlt eine vorausgelagerte Zeitungsleseforschung, die eine gesellschaftspolitische Leitfunktion fürs Zeitungslesen vorgeben könnte (Rühl, 2002).

Abschließend, als aktuelles Beispiel, der Begriff Kinderarmut in der gegenwärtigen Presse- und Rundfunkberichterstattung. Damit sind keine armen Kinder in Afrika oder im Kiez gemeint, und der Begriff Kinderarmut wird kategorial nicht präziser, fügt man ihm die Attribute kinderfeindlich, asozial oder eurozentrisch hinzu. Wer ständig Zeitung liest, kann wissen, dass Kinderarmut für einen „Bambini-Mangel“ in überalternden Gesellschaften steht, ein Sachverhalt mit makrosozialen Folgen und Folgeproblemen wie: geringeres Wirtschaftswachstum, niedrigere Steueraufkommen, steigende Probleme mit Abwanderung und Einwanderung, neuartige Formen der Literalität, Erziehung, Bildung, Ausbildung und so weiter. Würde eine Public Relations-Kampagne gegen Kinderarmut angesichts dieser sachlichen, sozialen und zeitlichen Widersprüche geplant werden, dann ist auf Kinderarmut aus inkongruenten Perspektiven einzugehen - auch wenn es scheinbar nur um Fundraising geht.

\section{Nochmals: Theorie der Allgemeinen Kommunikationswissenschaft}

Es gehört zum Alltagsgeschäft jeder Wissenschaft, Theorien unter wissenschaftsfähigen Bedingungen (Annahmen, Hypothesen, Metaphern, Begriffen, Modellen, Methoden, Daten) selbstkritisch zu vergleichen. Die vorliegende wissenschaftskritische Autopolemik aus der Kommunikationskommunität [communications community] enthält Grundzüge einer Theorie der Allgemeinen Kommunikationswissenschaft, die zur Diskussion anregen sollen. Werden dazu Aristoteles, Christian Thomasius, Kaspar Stieler, Adam Smith, Albert Schäffle, George Herbert Mead, Kenneth Burke, Harold D. Lasswell, Franz Ronneberger oder Niklas Luhmann zitiert, dann nicht als kommunikationswissenschaftliche Gründerväter oder als bislang unbeachtete Nestoren. Diese Namen stehen für Werke, die deshalb Klassiker sind, weil sie theoriefähige Lösungen für kommunikationswissenschaftliche Gegenwartsprobleme anbieten. Noch ist der Literaturhorizont für eine Allgemeine Kommunikationswissenschaft nicht zu standardisieren (Holtz-Bacha \& Kutsch, 2002), geschweige denn zu kanonisieren (Katz et al., 2002; zu beiden: Raabe, 2003). Eine Allgemeine Kommunikationswissenschaft kann kein deut- 
sches, auch kein amerikanisches Unternehmen sein, wenngleich jedes Kommunikationswissen durch die Grundoperationen Unterscheidung [distinction] und Bezeichnung [indication] zu axiomatisieren ist (Spencer Brown, 1997). In jedem einzelnen Lehroder Forschungsfall ist ein bestimmbarer Innenzustand (Bezugssystem) als Kommunikationseinheit zu markieren [marked space], der ein unbestimmter Kommunikationsaußenzustand [unmarked space] als Differenz gegenübersteht. Diese gleichzeitige Innen/Außen-Gesamtform wird „Gesetz“ für die Wahrnehmung. Mit den Beatles gesagt: „Your inside is out and your outside is in“ (Glanville/Varela, 1981).

Die Kommunikationswissenschaft kann kein fixes Kommunikationswissen besitzen, auch keine fixen empirischen Kontrollstandards, nicht einmal fixe Kommunikationserwartungen, auf die sie eindeutig reagieren kann. Stattdessen wird in und über Kommunikationssysteme selektierend, variierend und retentierend entschieden. Dennoch bleibt prozessiertes Kommunikationswissen kontingent, weil es sich als Wissen über Kommunikation durch Kommunikation selbst (relativ) konstituiert. „Only angels communicate absolutely“ (Burke, 1935/1965: XLIX). Insofern kann wissenschaftliches Kommunikationswissen als Summe jener Erklärungsversuche bezeichnet werden, die als Theorien bewabrt und erneuert werden.

Die Theorie einer Allgemeinen Kommunikationswissenschaft wird sich verabschieden müssen von den Ideen, mit Medien als Summenbegriff operieren oder Kommunikation als verdinglichte Ein-Weg-Verläufe modellieren zu können. Auch Information wird nicht, wie Phlogiston, ${ }^{2}$ der Kommunikation entweichen. Werden Medien-, Informations-, Erlebnis- oder Kommunikationsgesellschaften, (post-)industrielle, (post-) kapitalistische oder (neo-post-)kommunistische Gesellschaften, Dienstleistungs-, Risiko-, Armuts- oder Überflussgesellschaften im Zusammenhang mit Kommunikation aktiviert, dann werden gesellschaftliche Einzelaspekte hervorgehoben und Tendenzen angedeutet. Mit ausdrücklichen Innen-/Außen-Beziehungen von Kommunikation-/ Gesellschafts-Verhältnissen kann der Emergenz von Kommunikation jedoch gründlich nachgespürt werden, etwa der Erfindung von Symbolen und Sprachen, oder der heutigen „Koexistenz“ des phonetischen Schreibens (Skriptografie), des Vertextens durch den Buchdruck (Typografie) und der Umstrukturierung von Texten mithilfe elektronischer Techniken und Technologien (Elektronografie).

Es ist eine ärgerliche Tatsache, dass die Kommunikationswissenschaft wenig zu ihrer Selbstbeschreibung beiträgt. Sie kümmert sich um tausendundein Detail, vorrangig in der Annahme, „der Praxis“ unmittelbar dienen zu können. „Werch ein Illtum“ (Ernst Jandl). Die Kommunikationswissenschaft kann der Praxis von Familie, Politik, Wirtschaft, Religion, Kunst, Publizistik und anderen Funktionssystemen der Gesellschaft nicht als Superpraxis zu Diensten sein. Sie kann jedoch mit erkenntnistheoretischer und methodentheoretischer Hilfe kongruente Kommunikationsperspektiven problematisieren, reformulieren, methodengesteuert bearbeiten und hypothetisch wahr lösen. Das wiederum kann keine andere Kommunikationspraxis. Es ist zu erwarten, dass eine umbauende Theorie der Allgemeinen Kommunikationswissenschaft das Wissen über Kommunikation transparenter macht.

2 Mit Phlogiston bezeichnet die Parawissenschaft Alchemie eine Substanz, die beim Verbrennen regelmäßig entweichen soll. Phlogiston wurde nie nachgewiesen. Die Idee wird von der Chemie verworfen, die aus einer inkongruenten Perspektive nachweist, dass beim Verbrennen Sauerstoff binzutritt. 


\section{Literatur}

Anderson, James A. (1996): Communication theory. epistemological Foundations. New York, London: Guilford.

Aristoteles (1979): Nikomachische Ethik. Übers. und komm. v. Franz Dirlmeier. Darmstadt: Wissenschaftliche Buchgesellschaft.

Arnold, Carroll C. \& Bowers, John Waite (1984): Handbook of rhetorical and communication theory. Boston, London u. a.: Allyn and Bacon.

Bachelard, Gaston (1938/1978): Die Bildung des wissenschaftlichen Geistes. Beitrag zur Psychoanalyse der objektiven Erkenntnis. Frankfurt am Main: Suhrkamp.

Baumhauer, Otto A. (1986): Die sophistische Rhetorik. Eine Theorie sprachlicher Kommunikation. Stuttgart: Metzler.

Beckmanns Allgemeine Technologie (1783-1793/2002): Herrn Hofrath Beckmanns Vorlesungen über die Technologie. Vorgetragen zwischen den Jahren 1783 bis 1793. Eingel., komment. u. hrsg. von Alois Kernbauer. Graz: Akad. Druck- u. Verlagsanstalt.

Bentele, Günter \& Rühl, Manfred (Hrsg.) (1993): Theorien öffentlicher Kommunikation. Problemfelder, Positionen, Perspektiven. München: Ölschläger.

Berelson, Bernard (1952): Content analysis in communication research. Glencoe: Free Press.

Berlo, David K. (1960): The process of communication. An introduction to theory and practice. New York, Chicago u.a.: Holt, Rinehart and Winston.

Bonfadelli, Heinz \& Bucher, Priska (Hrsg.) (2002): Lesen in der Mediengesellschaft. Stand und Perspektiven der Forschung. Zürich: Pestalozzianum.

Bonfadelli, Heinz \& Rathgeb, Jürg (Hrsg.) (1997): Publizistikwissenschaftliche Basistheorien und ihre Praxistauglichkeit. Zürcher Kolloquium zur Publizistikwissenschaft (= Diskussionspunkt 33). Zürich: Seminar für Publizistikwissenschaft.

Burkart, Roland (1998): Kommunikationswissenschaft. Grundlagen und Problemfelder. Umrisse einer interdisziplinären Sozialwissenschaft. 3. Auflage. Wien, Köln: Böhlau.

Burke, Kenneth (1935/1965): Permanence and change. An anatomy of purpose. With an introduction by Hugh Dalziel Duncan. 2. Auflage. Indianapolis, New York: Bobbs-Merill.

Burke, Kenneth (1966): A dramatistic view of the origins of language and postscripts on the negative. In: ders.: Language as symbolic action. Essays on life, literature, and method. Berkeley, Los Angeles: University of California Press.

Cassirer, Ernst (1910/1990): Substanzbegriff und Funktionsbegriff. Untersuchungen über die Grundfragen der Erkenntniskritik. Darmstadt: Wissenschaftliche Buchgesellschaft.

Cooley, Charles Horton (1902/1964): Human nature and the social order. Introduction by Philip Rieff; Foreword by George Herbert Mead. 3. Auflage. New York: Schocken.

Cooley, Charles Horton (1909/1962): Social organization. A study of the larger mind. 3. Auflage. New York: Schocken.

Dædalus (1983): Themenheft „Scientific Literacy“, 112: 2.

Deutsche Gesellschaft für Publizistik- und Kommunikationswissenschaft (Hrsg.) (2001): Die Mediengesellschaft und ihre Wissenschaft. München: Selbstverlag.

Dovifat, Emil (Hrsg.) (1971): Handbuch der Publizistik. Bd. 1: Allgemeine Publizistik. 2. Auflage. Berlin: de Gruyter.

Dröge, Franz W. \& Lerg, Winfried B. (1965): Kritik der Kommunikationswissenschaft. In: Publizistik 10, 251-284.

Esser, Frank (2000): Journalismus vergleichen. Journalismustheorie und komparative Forschung. In: Löffelholz, Martin (Hrsg.): Theorien des Journalismus. Ein diskursives Handbuch. Wiesbaden: Westdeutscher Verlag, 123-145.

Glanville, Ranulph \& Varela, Francisco (1981): Your inside is out und your outside is in. In: Lasker, G. E. (Hrsg.): International Congress on Applied Systems Research and Cybernetics, Bd. 6. Oxford: Pergamon, 638-641

Groth, Otto (1913/1915): Die politische Presse Württembergs. Staatswissenschaftliche Diss. Tübingen; Stuttgart: Scheufele. 
Groth, Otto (1960): Die unerkannte Kulturmacht. Grundlegung der Zeitungswissenschaft (Periodik) Bde. 1. Berlin: de Gruyter.

Hardt, Hanno (1979): Social theories of the press. Early German \& American perspectives. Forword by James W. Carey. Beverley Hills, London: Sage.

Harman, David (1970): Illiteracy. An Overview. In: Harvard Educational Review 40, 226-243

Harman, David (1987): Illiteracy. A national dilemma. New York, Toronto: Cambridge Book Company.

Holtz-Bacha, Christina \& Kutsch, Arnulf (2002): Schlüsselwerke für die Kommunikationswissenschaft. Wiesbaden: Westdeutscher Verlag.

Innis, Harold A. (1950/1972): Empire and communications. Rev. by Mary Q. Innis. Forword by Marshall McLuhan. Toronto, Buffalo: Toronto UP.

Innis, Harold A. (1951/1982): The bias of communication. Introduction by Marshall McLuhan. Toronto, Buffalo: Toronto UP.

Jablin, Fredric M./Putnam, Linda L./Roberts, Karlene H. \& Porter, Lyman W. (Hrsg.) (1987): Handbook of organizational communication. An interdisciplinary perspective. Newbury Park u. a.: Sage.

Jahoda, Marie/Lazarsfeld, Paul F. \& Zeisel, Hans (1933/1975): Die Arbeitslosen von Marienthal. Ein soziologischer Versuch über die Wirkungen dauernder Arbeitslosigkeit. Mit einem Anhang zur Geschichte der Soziographie. 2. Auflage. Frankfurt am Main: Suhrkamp.

Kant, Immanuel (1788/1968): Kritik der praktischen Vernunft. Kant Werke. Bd. 6. Darmstadt: Wissenschaftliche Buchgesellschaft.

Kaplan, Abraham (1964): The conduct of inquiry. Methodology for behavioral science. Scranton: Chandler.

Katz, Elihu \& Lazarsfeld, Paul F. (1955): Personal influence. The part played by people in the flow of mass communication. New York: Free Press.

Katz, Elihu/Peters, John Durham/Liebes, Tamar \& Orloff, Avril (Hrsg.) (2002): Canonic texts in media research: Are there any? Should there be? How about these? Cambridge: Polity Press.

Kepplinger, Hans Mathias (2000): Problemdimensionen des Journalismus. Theoretischer Anspruch und empirischer Ertrag. In: Löffelholz, Martin (Hrsg.): Theorien des Journalismus. Ein diskursives Handbuch. Wiesbaden: Westdeutscher Verlag, 81-99.

Knies, Karl (1853): Die Eisenbahnen und ihre Wirkungen. Braunschweig: Schwetschke.

Knies, Karl (1857): Der Telegraph als Verkehrsmittel. Mit Erörterungen über den Nachrichtenverkehr überhaupt. Tübingen: Laupp.

Köcher, Renate (1985): Spürhund und Missionar. Eine vergleichende Untersuchung über Berufsethik und Aufgabenverständnis britischer und deutscher Journalisten. München: Phil. Diss.

Krippendorff, Klaus (1996): A second-order cybernetics of otherness. In: Systems Research 13, 311-328.

Kuhn, Thomas S. (1973): Die Struktur wissenschaftlicher Revolution. Frankfurt am Main: Suhrkamp.

Lasswell, Harold D. (1948/1987): The structure and function of communication in society. In: Bryson, Lyman (Hrsg.): The communication of ideas. New York: Cooper Square, 37-51. Nachdruck in: Gottschlich, Maximilian (Hrsg.): Massenkommunikationsforschung: Theorieentwicklung und Problemperspektiven. Wien: Braumüller, 17-26.

Lazarsfeld, Paul F. (1981): Mit Merton arbeiten. In: Lepenies, Wolf (Hrsg.): Geschichte der Soziologie. Studien zur kognitiven, sozialen und historischen Identität einer Disziplin. Bd. 1. Frankfurt am Main: Suhrkamp, 337-391.

Lazarsfeld, Paul F./Berelson, Bernard \& Gaudet, Hazel (1944/1968): The people's choice. How the voter makes up his mind in a presidential campaign. 3. Auflage. New York, London: Columbia University Press.

Löffelholz, Martin (Hrsg.) (2000): Theorien des Journalismus. Ein diskursives Handbuch. Wiesbaden: Westdeutscher Verlag.

Löffelholz, Martin (2003): Von 'neuen Medien' zu 'dynamischen Systemen'. Eine Bestandsaufnahme zentraler Metaphern zur Beschreibung der Emergenz öffentlicher Kommunikation. In: 
Altmeppen, Klaus-Dieter \& Karmasin, Matthias (Hrsg.): Medien und Ökonomie. Bd. 1/1: Grundlagen der Medienökonomie: Kommunikations- und Medienwissenschaft, Wirtschaftswissenschaft. Wiesbaden: Westdeutscher Verlag, 53-90.

Löffelholz, Martin \& Quandt, Thorsten (Hrsg.) (2003): Die neue Kommunikationswissenschaft. Theorien, Themen und Berufsfelder im Internet-Zeitalter. Eine Einführung. Wiesbaden: Westdeutscher Verlag.

Löffler, Franz Adam (1837): Ueber die Gesetzgebung der Presse. Ein Versuch zur Lösung ihrer Aufgabe auf wissenschaftlichem Wege. Erster Theil. Leipzig: Brockhaus.

Loosen, Wiebke/Scholl, Armin \& Woelke, Jens (2002): Systemtheoretische und konstruktivistische Methodologie. In: Scholl, Armin (Hrsg.): Systemtheorie und Konstruktivismus in der Kommunikationswissenschaft. Konstanz: UVK. 37-65.

Luhmann, Niklas (1965): Grundrechte als Institution. Ein Beitrag zur politischen Soziologie. Berlin: Duncker \& Humblot.

Luhmann, Niklas (1970): Soziologische Aufklärung. In: ders.: Soziologische Aufklärung. Aufsätze zur Theorie sozialer Systeme. Köln, Opladen: Westdeutscher Verlag, 66-91.

Luhmann, Niklas (1977): Arbeitsteilung und Moral. Durkheims Theorie. In: Durkheim, Emile: Über die Teilung der sozialen Arbeit (1893). Eingeleitet von Niklas Luhmann. Frankfurt am Main: Suhrkamp.

Luhmann, Niklas (1984): Soziale Systeme. Grundriss einer allgemeinen Theorie. Frankfurt am Main: Suhrkamp.

Luhmann, Niklas (1992): Die Wissenschaft der Gesellschaft. Frankfurt am Main: Suhrkamp.

Luhmann, Niklas (1995): Was ist Kommunikation? In: ders.: Soziologische Aufklärung 6. Opladen: Westdeutscher Verlag, 113-124.

Luhmann, Niklas (1997): Die Gesellschaft der Gesellschaft. 2 Bde., Frankfurt am Main: Suhrkamp.

Luhmann, Niklas (2000): Organisation und Entscheidung. Opladen, Wiesbaden: Westdeutscher Verlag.

Lynd, Robert S. \& Lynd, Helen M. (1929): Middletown. A study in American culture. New York: Hartcourt, Brace.

Lynd, Robert S. \& Lynd, Helen M. (1937): Middletown in transition. New York: Hartcourt, Brace.

Lynd, Robert S. (1939/1970): Knowledge for what? The place of social science in American culture. 2. Auflage. Princeton: Princeton UP.

Maletzke, Gerhard (1963): Psychologie der Massenkommunikation. Theorie und Systematik. 2. Auflage. Hamburg: Verlag Hans Bredow Institut.

McLuhan, Marshall (1969): Playboy Interview: Marshall McLuhan. In: Playboy, 53-74.

McLuhan, Marshall \& Fiore, Quentin (1967): The medium is the massage. New York: Bantom.

McQuail, Denis (1989): Mass communications research. In: International Encyclopedia of Communications. Bd. 2. New York, Oxford: Oxford University Press, 487-492.

McQuail, Denis \& Windahl, Sven (1998): Communication models for the study of communications. 2. Auflage. London, New York: Longman.

Mead, George Herbert (1934/1967): Mind, self, and society. From the standpoint of a social behaviorist. 2. Auflage. Chicago, London: Chicago UP.

Merten, Klaus (1995): Inhaltsanalyse: Einführung in Theorie, Methode und Praxis. 2. verb. Auflage. Opladen: Westdeutscher Verlag.

Merton, Robert K. (1957): Social theory and social structure. 2. überarb. und erw. Auflage. Glencoe: Free Press.

Obst, Bernhard (1986): Das Ende der Presse-Enquete Max WEBERs. Der Heidelberger Professorenprozess von 1912 und seine Auswirkungen auf die deutsche Zeitungswissenschaft. In: Bruch, Rüdiger vom \& Roegele, Otto B. (Hrsg.): Von der Zeitungskunde zur Publizistik. Biographisch-institutionelle Stationen der deutschen Zeitungswissenschaft in der ersten Hälfte des 20. Jahrhunderts. Frankfurt am Main: Haag + Herchen Verlag, 45-62.

Ortmann, Günther/Sydow, Jörg \& Türk, Klaus (Hrsg.) (1997): Theorien der Organisation. Die Rückkehr der Gesellschaft. Opladen: Westdeutscher Verlag.

Parsons, Talcott (1973): Culture and social system revisited. In: Schneider, Louis \& Bonjean, 
Charles M. (Hrsg.): The idea of culture in the social sciences. London: Cambridge University Press, 33-46.

Pöttker, Horst (2000): Kompensation und Komplexität. Journalismustheorie als Begründung journalistischer Qualitätsmaßstäbe. In: Löffelholz, Martin (Hrsg.): Theorien des Journalismus. Wiesbaden: WV, 101-122.

Prakke, Henk/Dröge, Franz W./Lerg, Winfried B. \& Schmolke, Michael (1968): Kommunikation der Gesellschaft. Einführung in die funktionale Publizistik. Münster: Regensberg.

Prutz, Robert E. (1845/1971): Geschichte des deutschen Journalismus. Erster Teil. 2. Auflage. Göttingen: Vandenhoeck \& Ruprecht.

Raabe, Johannes (2003): Buchbesprechungen - Essay. In: Publizistik, 48, 357-361.

Riepl, Wolfgang (1913/1972): Das Nachrichtensystem des Altertums, mit besonderer Rücksicht auf die Römer. 2. Auflage. Hildesheim, New York: Olms.

Rogers, Everett M. \& Chaffee, Steven H. (1994): Communication and journalism from „Daddy“ Bleyer to Wilbur Schramm. A palimpsest. Journalism Monographs 148. Columbia : AEJMC.

Rogers, Everett M. (1994): A history of communication study. A biographical approach. New York: Free Press; Oxford, Singapore, Sidney: Maxwell Macmillan.

Ronneberger, Franz (1964/1974): Die politischen Funktionen der Massenkommunikationsmittel. In: Publizistik, 9, 291-304. Nachdruck in: Langenbucher, Wolfgang R. (Hrsg.): Zur Theorie der politischen Kommunikation. München: Piper, 193-205.

Ronneberger, Franz (1970): Was Kommunikationsforschung mit Politik zu tun hat. In: Deutsche Gesellschaft für Publizistik- und Zeitungswissenschaft (Hrsg.): Publizistik - Zeitungswissenschaft - Communication Research - Journalism. Dokumentation, dt. und engl. Konstanz: Universitätsverlag, 60-67.

Ronneberger, Franz (1971): Sozialisation durch Massenkommunikation. In: ders. (Hrsg.): Sozialisation durch Massenkommunikation. Stuttgart: Enke, 32-101.

Ronneberger, Franz (1978-1986): Kommunikationspolitik I-III. Mainz: v. Hase \& Koehler.

Rössler, D. (1980): Mensch, ganzer. In: Ritter, Joachim \& Gründer, Karlfried (Hrsg.): Historisches Wörterbuch der Philosophie. Bd. 5. Darmstadt: Wissenschaftliche Buchgesellschaft, 1106-1111.

Rühl, Manfred (1969/1979): Die Zeitungsredaktion als organisiertes soziales System. Überarb. und erw. 2. Auflage Fribourg: Universitätsverlag.

Rühl, Manfred (1980): Journalismus und Gesellschaft. Bestandsaufnahme und Theorieentwurf. Mainz: v. Hase und Koehler.

Rühl, Manfred (1985): Kommunikationswissenschaft zwischen Wunsch und Machbarkeit. Einige Betrachtungen zu ihrer Identität heute. In: Publizistik, 30, 229-246.

Rühl, Manfred (1986): Ordnungspolitische Probleme eines künftigen Rundfunks in der Bundesrepublik Deutschland. In: Fleck, Florian H. (Hrsg.): Zukunftsaspekte des Rundfunks. Stuttgart: Kohlhammer, 77-101.

Rühl, Manfred (1987): Humankommunikation und menschliche Erfahrung. Zum Umbau von Kernbegriffen in der gegenwärtigen Gesellschaft. In: ders. (Hrsg.): Kommunikation und Erfahrung. Wege anwendungsbezogener Kommunikationsforschung. Nürnberg: Verlag der Kommunikationswissenschaftlichen Forschungsvereinigung, 5-66.

Rühl, Manfred (1988): Zur Technisierung freiheitlicher Publizistik - jenseits von Neuen Medien und Neuer Technik. In: Bungard, Walter \& Lenk, Hans (Hrsg.): Technikbewertung. Philosophische und psychologische Perspektiven. Frankfurt am Main: Suhrkamp, 343-377.

Rühl, Manfred (1989/2003): Organisatorischer Journalismus. Tendenzen der Redaktionsforschung. In: Kaase, Max \& Schulz, Winfried (Hrsg.): Massenkommunikation. Theorien, Methoden, Befunde (= Sonderheft 30 der Kölner Zeitschrift für Soziologie und Sozialpsychologie). Opladen: Westdeutscher Verlag, 252-269. Nachdruck in: Neverla, Irene/Grittmann, Elke \& Pater, Monika (Hrsg.): Grundlagentexte zur Journalistik. Konstanz: UVK, 303-320.

Rühl, Manfred (1993): Marktpublizistik. Oder: Wie alle - reihum - Presse und Rundfunk bezahlen. In: Publizistik 38, 125-152.

Rühl, Manfred (1998): Von fantastischen Medien und publizistischer Medialisierung. In: Dernbach, Beatrice/Rühl, Manfred \& Theis-Berglmair, Anna Maria (Hrsg.): Publizistik im vernetzten 
Zeitalter. Berufe - Formen - Strukturen. Opladen/Wiesbaden: Westdeutscher Verlag, 95107.

Rühl, Manfred (1999): Publizieren. Eine Sinngeschichte der öffentlichen Kommunikation. Opladen, Wiesbaden: Westdeutscher Verlag.

Rühl, Manfred (2000a): Des Journalismus vergangene Zukunft. Zur Theoriegeschichte einer künftigen Journalismusforschung. In: Löffelholz, Martin (Hrsg.): Theorien des Journalismus. Ein diskursives Handbuch. Wiesbaden: Westdeutscher Verlag, 65-79.

Rühl, Manfred (2000b): Technik und ihre publizistische Karriere. In: Jarren, Otfried/Kopper, Gerd G. \& Toepser-Ziegert, Gabriele (Hrsg.): Zeitung - Medium mit Vergangenheit und Zukunft. Eine Bestandsaufnahme (= Festschrift aus Anlass des 60. Geburtstages von Hans Bohrmann). München: Saur, 93-104.

Rühl, Manfred (2000c): Medien (alias Mittel) und die öffentliche Kommunikation. Ein alteuropäisches Begriffspaar im Wirklichkeitswandel. In: Zurstiege, Guido (Hrsg.): Festschrift für die Wirklichkeit. Wiesbaden: Westdeutscher Verlag, 105-118.

Rühl, Manfred (2001): Alltagspublizistik. Eine kommunikationswissenschaftliche Wiederbeschreibung. In: Publizistik 46, 249-276.

Rühl, Manfred (2002): Zeitunglesen und die Lesbarkeit der Welt. In: Bonfadelli, Heinz \& Bucher, Priska (Hrsg.): Lesen in der Mediengesellschaft. Stand und Perspektiven der Forschung. Zürich: Verlag Pestalozzianum, 82-96.

Rühl, Manfred (2003): Politische Ökonomie der Alltagspublizistik. Suchen und Prüfen von Grundlagen für ein Theorieprogramm. In: Altmeppen, Klaus-Dieter \& Karmasin, Matthias (Hrsg.): Medien und Ökonomie. Bd. 1/1: Grundlagen der Medienökonomie: Kommunikations- und Medienwissenschaft, Wirtschaftswissenschaft. Wiesbaden: Westdeutscher Verlag, 91-114.

Rühl, Manfred (2004a): Für Public Relations? Ein kommunikationswissenschaftliches Theorienbouquet! In: Röttger, Ulrike (Hrsg.): Theorien der Public Relations. Wiesbaden: Westdeutscher Verlag (im Druck).

Rühl, Manfred (2004b): Des Journalismus vergangene Zukunft. Zur Emergenz der Journalistik. In: Löffelholz, Martin: Theorien des Journalismus. Ein diskursives Handbuch. 2. Auflage. Wiesbaden: Westdeutscher Verlag (im Druck).

Ruhrmann, Georg/Kohring, Matthias/Görke, Alexander/Maier, Michaela \& Woelke, Jens (2000): Im Osten was Neues? Ein Beitrag zur Standortbestimmung der Kommunikations- und Medienwissenschaft. In: Publizistik 45, 282-309.

Rusch, Gebhard (Hrsg.) (2002): Einführung in die Medienwissenschaft. Konzeptionen, Theorien, Methoden, Anwendungen. Wiesbaden: Westdeutscher Verlag.

Saxer, Ulrich (1999): Die Medienwissenschaft I: Grundlagen, 1. Der Forschungsgegenstand der Medienwissenschaft. In: Leonhard, Joachim-Felix/Ludwig, Hans-Werner/Schwarze, Dietrich \& Straßner, Erich (Hrsg.): Medienwissenschaft. Ein Handbuch zur Entwicklung der Medien und Kommunikationsformen. 1. Teilband. Berlin, New York: de Gruyter, 1-14.

Schäffle, Albert (1875-1878): Bau und Leben des socialen Körpers. 4 Bde. Tübingen: Laupp 1875-1878.

Schlözer, August Ludwig (1777): Entwurf zu einem Reise-Collegio, nebst einer Anzeige seines Zeitungs-Collegii. Göttingen: Vandenhoek.

Scholl, Armin (Hrsg.) (2002): Systemtheorie und Konstruktivismus in der Kommunikationswissenschaft. Konstanz: UVK.

Scholl, Armin \& Weischenberg, Siegfried (1998): Journalismus in der Gesellschaft. Theorie, Methodologie und Empirie. Opladen, Wiesbaden: Westdeutscher Verlag.

Schulz, Winfried (Hrsg.) (1986): Medienwirkungsforschung in der Bundesrepublik Deutschland. Teil I: Berichte und Empfehlungen. Teil II: Dokumentation. Katalog der Studien. Wenham: $\mathrm{VCH}$.

Shannon, Claude E. \& Weaver, Warren (1949): The mathematical theory of communication. Urbana, Chicago, London: University of Illinois Press.

Smith, Adam (1759/1976): The theory of moral sentiments. Edited by D. D. Raphael; Macfie, A. L. Oxford: Clarendon Press.

Smith, Adam (1776/1974): Der Wohlstand der Nationen. Eine Untersuchung seiner Natur und sei- 
ner Ursachen. Aus dem Englischen übertragen mit einer Würdigung von Horst Claus Recktenwald. München: Beck.

Solomon, Howard M. (1972): Public welfare, science, and propaganda in Seventeenth Century France. The innovations of Théophraste Renaudot. Princeton: Princeton UP.

Spencer-Brown, George (1997): Laws of Form - Gesetze der Form. Lübeck: Bohmeier.

Stieler, Kaspar (1695/1969): Zeitungs Lust und Nutz. Vollständiger Neudruck der Orginalausgabe von 1695, hrsg. von Gert Hagelweide. 2. Auflage. Bremen: Schünemann.

Theis, Anna Maria (1994/2003): Organisationskommunikation. Theoretische Grundlagen und empirische Forschungen. Opladen: Westdeutscher Verlag.

Thomasius, Christian (1692/1994): Die neue Erfindung einer wohlgegründeten und für das gemeine Wesen höchstnöthigen Wissenschaft. In: Kleine teutsche Schriften [= Ausgewählte Werke, Bd. 22]. Neudruck Hildesheim u.a.: Olms, 449-490.

Thomasius, Christian (1692/1995): Einleitung zur Sittenlehre [Von der Kunst Vernünfftig und Tugenhafft zu lieben. Als dem eintzigen Mittel zu einer glückseligen/galanten und vergnügten Leben zu gelangen/oder Einleitung zur SittenLehre. Vorwort von Werner Schneiders [= Ausgewählte Werke, Bd. 10] Nachdruck: Hildesheim, Zürich, New York: Olms.

Walter-Busch, Emil (1996): Organisationstheorien von Weber bis Weick. Amsterdam: OPA.

Weaver, Warren (1949): Recent contributions to the mathematical theory of communication. In: Shannon, Claude E. \& Weaver, Warren: The mathematical theory of communication. Urbana: University of Illinois Press, 1-28.

Weber, Max (1922/1985): Wirtschaft und Gesellschaft. Grundriß der verstehenden Soziologie, hrsg. v. Johannes Winckelmann. Tübingen: Mohr (Siebeck).

Weick, Karl E. (1995): Sensemaking in organizations. Thousand Oaks, London, New Delhi: Sage.

Wettstein, Oscar (1907): Das jüngste Kind der Universität. In: Meissner, J. Friedrich (Hrsg.): Studien über das Zeitungswesen. Professor Dr. Adolf Koch, dem Begründer und Leiter des journalistischen Seminars der Universität Heidelberg anläßlich der Vollendung des 20. Seminar-Semesters gewidmet von seinen Schülern und Freunden. Frankfurt am Main: Meissner, 3-11.

Wiebe, Gerhart D. (1955): Massenkommunikationen. In: Hartley, Eugene L. \& Hartley, Ruth E.: Die Grundlagen der Sozialpsychologie, 109-137.

Wright, Charles R. (1959/1986): Mass communication. A sociological perspective. 3. Auflage. New York: Random House. 